\title{
Effects of Cyclocarya paliurus
} Aqueous and Ethanol Extracts on Glucolipid Metabolism and the Underlying Mechanisms: A Meta-Analysis and Systematic Review

OPEN ACCESS

Edited by:

Omotayo Erejuwa

Ebonyi State University, Nigeria

Reviewed by:

Chengyuan Lin,

Hong Kong Baptist University,

Hong Kong

Makan Pourmasoumi,

Guilan University of Medical

Sciences, Iran

${ }^{*}$ Correspondence:

Lili Wu

qingniao_566@163.com

Tonghua Liu

thliu@vip.163.com

†These authors have contributed equally to this work and share first authorship

Specialty section:

This article was submitted to Nutrition and Metabolism,

a section of the journa

Frontiers in Nutrition

Received: 12 September 2020 Accepted: 10 November 2020 Published: 01 December 2020

Citation:

Liu W, Wu Y, Hu Y, Qin S, Guo X, Wang M, Wu L and Liu T (2020) Effects of Cyclocarya paliurus

Aqueous and Ethanol Extracts on

Glucolipid Metabolism and the

Underlying Mechanisms: A Meta-Analysis and Systematic Review.

Front. Nutr. 7:605605

doi: 10.3389/fnut.2020.605605

\begin{abstract}
Wei Liu ${ }^{1,2 t}$, You Wu ${ }^{1,2 t}$, Yuli Hu ${ }^{1,2}$, Shuai Qin ${ }^{3}$, Xiaoyuan Guo ${ }^{2}$, Minghui Wang ${ }^{4}$, Lili Wu ${ }^{1 *}$ and Tonghua Liu ${ }^{1 *}$

${ }^{1}$ Key Laboratory of Health Cultivation of the Ministry of Education, Beijing University of Chinese Medicine, Beijing, China, 2 Dongfang Hospital, Beijing University of Chinese Medicine, Beijing, China, ${ }^{3}$ First School of Clinical Medicine, Guizhou University of Traditional Chinese Medicine, Guiyang, China, ${ }^{4}$ Chengdu Integrated TCM and Western Medicine Hospital, Chengdu, China
\end{abstract}

Background and Aims: Cyclocarya paliurus (CP) has been used as an herbal tea to treat diabetes mellitus and obesity for hundreds of years. Previous research suggests that CP specifically restores glucolipid metabolic homeostasis, and the two most studied preparations are aqueous and ethanol extracts. In order to verify the effect of CP on glucolipid metabolism in animal models with metabolic syndrome, a meta-analysis was performed, and the active components and underlying mechanisms were systematically reviewed.

Methods: Four databases: PubMed, Web of Science, Embase, and Cochrane Library were searched to identify potential literature. Data of blood glucose (BG) level, area under curve (AUC) of oral glucose tolerance test (OGTT), total cholesterol (TC), triglyceride (TG), high-density lipoprotein (HDL), and low-density lipoprotein (LDL) levels were extracted as indicators of the assessment of CP's effects. Follow-up analyses including subgroup analysis, meta-regressions, and publication bias were also conducted.

Results: A total of 96 papers were identified from the databases and 11 papers including 31 data reports were involved in the meta-analysis. CP had a positive effect in down-regulating BG, AUC of OGTT, TC, TG, and LDL, and up-regulating HDL $(P<0.001$, $95 \%$ confidence interval of standard mean difference did not incorporate the null value 0 ).

Conclusion: CP showed definite activity of regulating glucolipid metabolism in animal models, and it exerted its function through multiple mechanisms including but not limited to: (1) improving insulin resistance; (2) protecting pancreatic $\beta$ cells; (3) decreasing inflammatory infiltration; and (4) anti-oxidative stress.

Keywords: Cyclocarya paliurus, metabolic syndrome, insulin resistance, dyslipidemia, hyperglycemia, glucolipid metabolism 


\section{INTRODUCTION}

Metabolic syndrome, also known as syndrome $\mathrm{X}$, is a complex syndrome characterized by glucose intolerance, insulin resistance, visceral obesity, dyslipidemia, and hypertension (1). It is associated with the global prevalence of diabetes mellitus and contributes to the increasing risk of cardiovascular diseases (2). The incidence of metabolic syndrome runs parallel with, but is three times more prevalent than that of type 2 diabetes, so it is estimated to influence around $25 \%$ of the global population (3).

The definition of metabolic syndrome has varied since the concept was established (4). However, in 2009, a harmonized standard was formed by five organizations (5), and this united criteria included the followed parameters: waist circumference, fasting plasma glucose level, high-density lipoprotein cholesterol (HDL-C) level, triglyceride (TG) level, and blood pressure level. Over-consumption of energy and lack of exercise are believed to be the predominant causes of metabolic syndrome. The accumulation of visceral fat is related to insulin resistance, glucose intolerance, lower HDL-C level, and higher low-density lipoprotein cholesterol (LDL-C) level and TG levels (6). So, there is no doubt that the regulation of glucolipid metabolism plays an important role in controlling metabolic syndrome.
Management strategies for metabolic syndrome are yet to be developed, and lifestyle modification and drug therapy are currently considered to be the two main approaches for its control (1). Cyclocarya paliurus (CP) (Batal.) Iljinsk (family Cyclocaryaceae) is an indigenous plant distributed throughout Southern China, the leaves of which have been used as an herbal tea to treat hyperglycemia and obesity in the local population (7). Recent scientific research shows that CP acts to reduce blood sugar levels and blood lipid levels, lowers body weight, and lowers blood pressure (8). Furthermore, a health tea made from CP leaves was approved by the United States Food and Drug Administration (FDA) in 1999, and was the first Chinese herbal tea approved by the institution $(9,10)$. In short, as a dietary supplement, CP seems to be a potential candidate for assisting in the prevention and treatment of metabolic syndrome.

To date, studies on CP have mostly involved preclinical research based on rodents. Among these studies, aqueous extract and ethanol extract have been the two most commonly studied CP preparations. Because of discrepancies in extraction processes, the components of the two extracts are different: polysaccharides are abundant in the aqueous extract (10), while the ethanol extract is enriched with flavonoids and triterpenoids (11). Polysaccharides (12), triterpenic acids (13, 14),

TABLE 1 | Characteristics of articles involved in the meta-analysis.

\begin{tabular}{|c|c|c|c|c|c|c|c|c|}
\hline Study & Country & Extract & Animal model & Gender & $\begin{array}{l}\text { Number per } \\
\text { group }\end{array}$ & Dose(mg/kg) & $\begin{array}{l}\text { Duration } \\
\text { (weeks) }\end{array}$ & Outcome \\
\hline Jiang et al. (31) & China & $\begin{array}{l}\text { Ethanol, } \\
\text { Aqueous }\end{array}$ & $\mathrm{KM}$ mice + HFD & Male & 10 & $\begin{array}{l}\text { Ethanol 1,500 } \\
\text { Aqueous 1,500 }\end{array}$ & 10 & $\begin{array}{l}\text { TC, TG, HDL, } \\
\text { LDL }\end{array}$ \\
\hline $\begin{array}{l}\text { Kurihara et al. } \\
\text { (32) }\end{array}$ & Japan & Aqueous & $\mathrm{KK}-\mathrm{A}^{\mathrm{y}}$ & Male & 11 & 2,000 & 3 & $B G$ \\
\hline Li et al. (33) & China & Aqueous & $\begin{array}{l}\text { SD rats + HFD + STZ } \\
(35 \mathrm{mg} / \mathrm{kg})\end{array}$ & Male & 6 & 200 & 12 & $\mathrm{BG}, \mathrm{TC}, \mathrm{TG}$ \\
\hline Liu et al. (15) & China & $\begin{array}{l}\text { Ethanol, } \\
\text { Aqueous }\end{array}$ & $\begin{array}{l}\text { C57BL } / 6 \text { mice + HFD } \\
+\mathrm{STZ}(40 \mathrm{mg} / \mathrm{kg})\end{array}$ & Male & 6 & $\begin{array}{l}\text { Ethanol 8,000 (5 } \\
\text { groups) } \\
\text { Aqueous 8,000 } \\
\text { (5 groups) }\end{array}$ & 4 & $\begin{array}{l}\text { BG, TC, TG, } \\
\text { HDL, LDL, } \\
\text { OGTT }\end{array}$ \\
\hline Ma et al. (11) & China & Ethanol & $\mathrm{KM}$ mice + HFD & Male & 8 & $\begin{array}{l}\text { Ethanol } \\
370 / 750 / 1,500\end{array}$ & 4 & $\begin{array}{l}\text { TC, TG, HDL, } \\
\text { LDL }\end{array}$ \\
\hline $\begin{array}{l}\text { Sheng et al. } \\
(17)\end{array}$ & China & Aqueous & $\begin{array}{l}\text { SD rats + HFD + } \\
\text { alloxan }(105 \text { mg/kg) }\end{array}$ & Male & 8 & Aqueous 1,000 & 5 & $\begin{array}{l}\text { BG, TC, TG, } \\
\text { HDL, LDL, } \\
\text { OGTT }\end{array}$ \\
\hline $\begin{array}{l}\text { Wang et al. } \\
(16)\end{array}$ & China & $\begin{array}{l}\text { Ethanol, } \\
\text { Aqueous }\end{array}$ & $\begin{array}{l}\text { SD rats + HFD + 5\% } \\
\text { sucrose-containing } \\
\text { water + STZ ( } 30 \\
\mathrm{mg} / \mathrm{kg})\end{array}$ & Male & 6 & $\begin{array}{l}\text { Ethanol } \\
\text { 2,000/4,000/8,000 } \\
\text { Aqueous 2,000/4,00 }\end{array}$ & $\begin{array}{r}4 \\
0 / 8,000\end{array}$ & $\begin{array}{l}\text { BG, TC, TG, } \\
\text { HDL, LDL }\end{array}$ \\
\hline $\begin{array}{l}\text { Wang et al. } \\
\text { (34) }\end{array}$ & China & Aqueous & $\begin{array}{l}\text { Wistar rats + HFD + } \\
\text { STZ }(35 \mathrm{mg} / \mathrm{kg})\end{array}$ & Male & $\begin{array}{c}\text { Model:13 } \\
\text { Low dose: } 9 \\
\text { High dose: } 11\end{array}$ & Aqueous 47/94 & 8 & $B G$ \\
\hline Xiao et al. (9) & China & Aqueous & $\begin{array}{l}\text { C57/BL6J mice + HFD } \\
+ \text { STZ }(25 \mathrm{mg} / \mathrm{kg})\end{array}$ & Male & 8 & $\begin{array}{l}\text { Aqueous } \\
1,000 / 2,000 / 4,000\end{array}$ & 5 & $\begin{array}{l}\text { BG, TC, TG, } \\
\text { HDL, LDL, } \\
\text { OGTT }\end{array}$ \\
\hline Xu et al. (10) & China & Aqueous & SHR/cp rats & Male & 6 & Aqueous 500 & 7 & $\mathrm{BG}, \mathrm{TC}, \mathrm{TG}$ \\
\hline Zhao et al. (8) & China & Ethanol & $\begin{array}{l}\text { C57BL/6J mice + HFD } \\
+ \text { STZ }(40 \mathrm{mg} / \mathrm{kg})\end{array}$ & $\begin{array}{l}\text { Male and } \\
\text { Female }\end{array}$ & 5 & Ethanol 500 & 4 & BG, OGT \\
\hline
\end{tabular}

BG, blood glucose level; HDL, high-density lipoprotein; LDL, low-density lipoprotein; OGT, oral glucose tolerance test; TC, total cholesterol; TG, triglycerides. 
and flavonoids (15) were initially believed to be the active constituents of $\mathrm{CP}$, however, there is also a study suggesting that polysaccharides may not be the anti-diabetic component of CP (16). Moreover, some studies also suggest that Cyclocarya paliurus aqueous extract (CPAE) shows no significant impact on lipid metabolic profile $(15,17)$. This controversy could be answered by a meta-analysis that would combine current evidence and reach a more robust conclusion. Moreover, previous studies have found that CP exerts a protective effect over pancreatic $\beta$ cells (8), has an anti-inflammatory effect $(7,18)$, and an anti-oxidant effect $(16,19)$ in animal models; however, the detailed mechanisms of CP's therapeutic activities still need to be unveiled.

In the current study, we performed a meta-analysis regarding the effect of CPAE and Cyclocarya paliurus ethanol extract (CPEE) on glucolipid metabolism with data obtained from preclinical research. Blood glucose (BG) level and the area under curve (AUC) of the oral glucose tolerance test (OGTT) are viewed as indicators of a hypoglycemic effect, while total cholesterol (TC), triglyceride (TG), high-density lipoprotein (HDL), and low-density lipoprotein (LDL) are used as indicators of a hypolipidemic effect. A systematic review was performed on the mechanisms studied by previous researchers, and we also summarized the current extraction methods for $\mathrm{CP}$, and the major components of CPAE and CPEE, so as to form a comprehensive understanding of presently available articles and provide an inclusive reference for further studies.

\section{METHODS}

This meta-analysis and systematic review were conducted according to Preferred Reporting Items for Systematic Reviews and Meta-Analyses (The PRISMA Statement) (20).

\section{Search Strategy}

Four academic databases: PubMed, Web of Science, Embase, and Cochrane Library, were searched from inception to May 2020. The PICO (patients, interventions, comparisons, outcomes) adopted in this analysis were as follows: (1) Animal models of T2DM/obesity/hyperlipidemia/metabolic syndrome. (2) CP extracts were orally administered to the animals and interventions were not combined with other chemicals or treatments. (3) The comparison group consisted of a same model that was treated with a placebo. (4) BG, OGTT, TC, TG, HDL, or LDL for the treatment and comparison groups at the end of intervention. In order to find the required

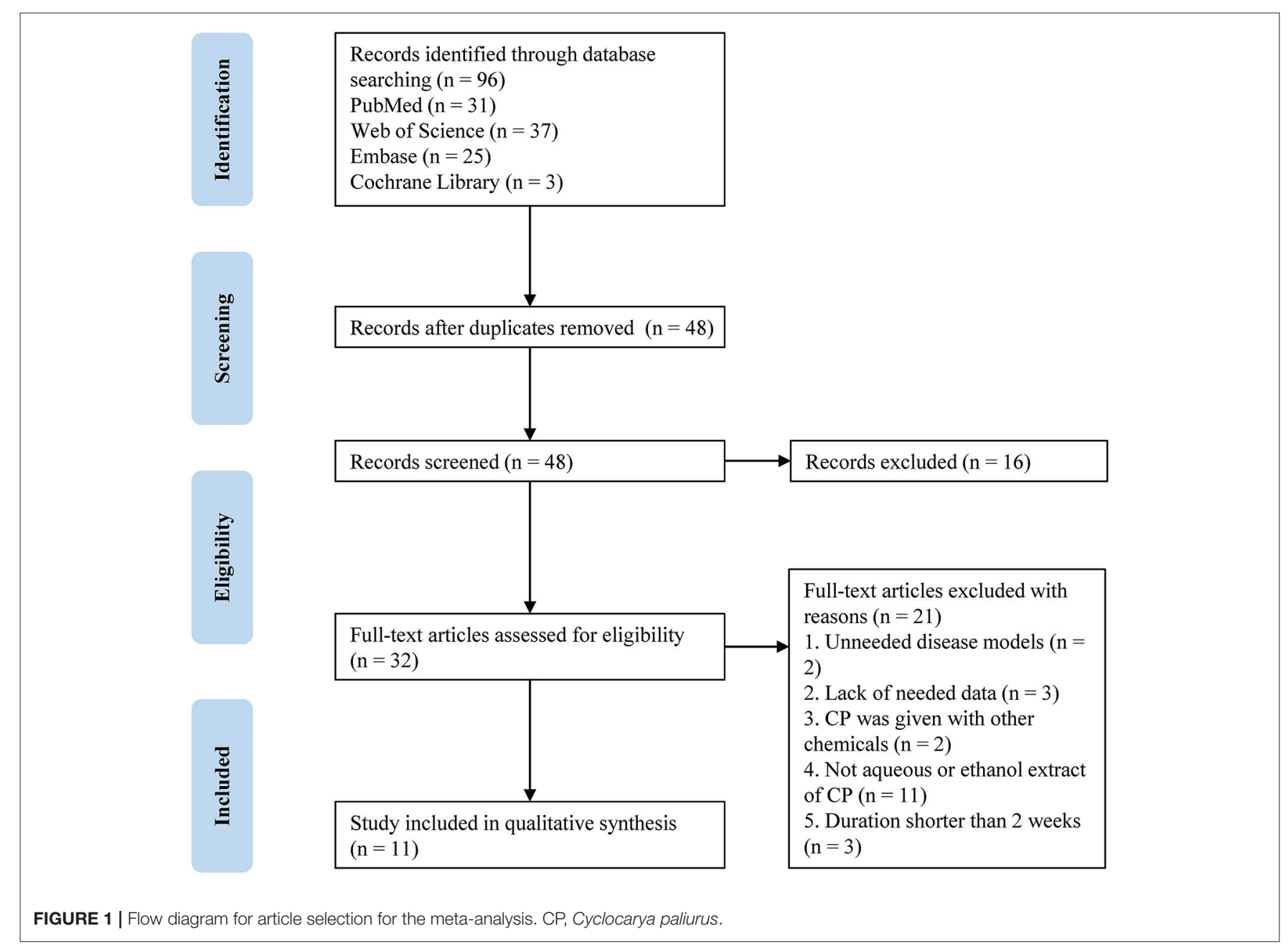


articles, searches were conducted as follows: (1) search "Cyclocarya" or "sweet tea tree" (title/abstract/keywords/MeSH); (2) search diseases with the following terms: "diabetes," "diabetic," "insulin resistance," "obesity," "hyperlipidemia," and "glucose intolerance" (title/abstract/keywords/MeSH); (3) terms, such as "animal," "rat," "mouse," and "rodent" (title/abstract/keywords/MeSH) were used to discover animal experiments; (4) the search queries above were combined together using "AND” (Supplementary Table 1). There was no limit on language, study design, and type of publication adopted for the research results.

\section{Eligibility Criteria for Studies Inclusion Criteria}

The inclusion criteria for the title and abstract were as follows: (1) not a review/protocol/letter/meta-analysis/proceeding; (2) oral administration of CP extract or component (no combination with other chemicals or treatments); (3) animal experiments (neither clinical trials nor in vitro research); (4) animal models of T2DM/obesity/hyperlipidemia/metabolic syndrome.

\section{Exclusion Criteria for Systematic Review}

The exclusion criteria for meta-analysis were as follows: (1) lack of full-text; (2) unneeded disease model; (3) not aqueous or ethanol extract of CP.

\section{Exclusion Criteria for Meta-Analysis}

The exclusion criteria for meta-analysis were as follows: (1) lack of full-text; (2) unneeded disease model; (3) not aqueous or ethanol extract of CP; (4) lack of data required; (5) treatment that lasted for $<2$ weeks; (6) repeated data in more than one article; (7) inconsistent depiction in article.

The titles and abstracts of articles from search results were examined by two independent researchers, duplicates were eliminated, and studies that met the inclusion criteria were reserved. Then researchers screened the full-text of articles according to the exclusion criteria, and the remaining articles were considered eligible. Whenever there were disagreements, a third author was consulted.

\section{Data Extraction}

The following data was extracted by two independent researchers: (1) publication information: first author's name, title, country, year of publication; (2) animal model information: animal strain, gender, age/body weight, number per group, model establishments; (3) administration information: dose, duration, CP preparations (aqueous or ethanol).

Outcome measurements were as follows: blood glucose level $(\mathrm{mg} / \mathrm{dL})$, AUC of OGTT $\left(\mathrm{mmol}^{*} \mathrm{~L}^{-1} * \mathrm{~h}\right)$, TC $(\mathrm{mg} / \mathrm{dL})$, TG $(\mathrm{mg} / \mathrm{dL}), \mathrm{HDL}(\mathrm{mmol} / \mathrm{L}), \mathrm{LDL}(\mathrm{mmol} / \mathrm{L})$ all expressed as mean \pm standard deviation (SD). Data presented in graphs were extracted by Web-plot Digitizer software from https:// automeris.io/WebPlotDigitizer/. Data from different dosages in one article were treated as separate data reports. Questions regarding the data were managed by sending E-mails to the corresponding authors.

\section{Assessment of Risk of Bias}

The risk of bias and quality of eligible articles was assessed by SYRCLE's risk of bias tool for animal studies (21). The SYRCLE tool is currently the most commonly used assessment tool that is specifically aimed at measuring the risk of bias in animal experiments. It contains 10 items that represent different aspects of risk of bias. Final results of the evaluation are expressed as "low risk," "high risk," or "unclear risk." According to the instructions, the results of assessment should be presented in a table or chart. In the current study, two researchers evaluated the risk of bias independently after training. Disaccords were resolved by discussion.

\section{Meta-Analysis}

Meta-analyses in this article were performed using RevMan 5.3 and STATA 14.0 software. The effect size was expressed as the standardized mean difference (SMD) with a 95\% confidence interval (CI). $P<0.05$ was considered as statistically significant. All data were calculated using a random effects model and using Hedges' method.

\section{Assessment and Identification of Heterogeneity}

In order to quantify the influence of heterogeneity on metaanalysis, a Higgins' $\mathrm{I}^{2}$ test (22) was conducted to examine the heterogeneity between eligible articles. $\mathrm{I}^{2}$ ranges from 0 to $100 \%$ indicated no heterogeneity up to a high potential of heterogeneity.

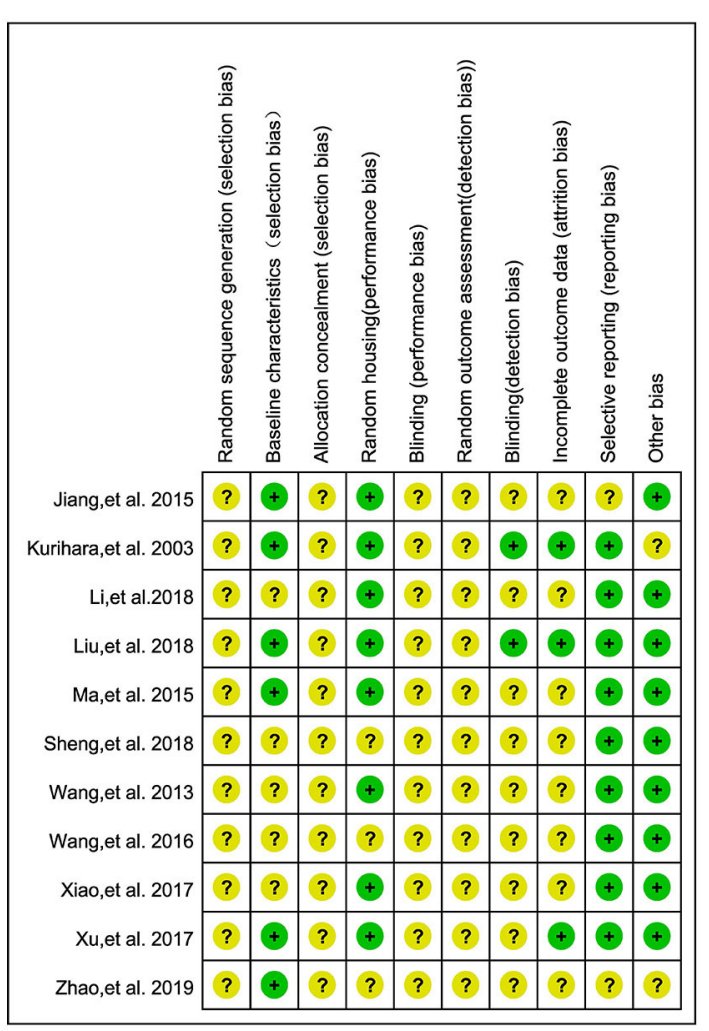

FIGURE 2 | Risk of bias of 11 studies included in the meta-analysis. Yellow dots, unclear risk; green dots, low risk. 
To identify the source of possible heterogeneity, subgroup analysis, meta-regression, and sensitivity tests were conducted based on different factors. Each outcome was divided into subgroups according to extract (AE, EE), and dose $(<1,000$, $1,000-2,000,4,000,8,000 \mathrm{mg} / \mathrm{d})$. In addition, meta-regression was performed according to the same factors. Meta-regression detects the correlation of each factor (extract, dose, duration, model generation) to outcomes, $P<0.05$ indicated high relevance between a studied factor and the outcome. In the metaregression analyses, dose and duration of treatment were treated as quantitative variables and different animal models and extracts were treated as dummy variables (non-quantitative variables). Furthermore, sensitivity tests were performed by removing those studies with a high risk of bias from the meta-analysis.

\section{Assessment of Publication Bias}

Since articles with positive results are more likely to be accepted and published, results of meta-analyses might be affected by publication bias. Therefore, a funnel plot was conducted to visually assess the publication bias. The estimation of effect size from each included study was plotted against a measure of precision (standard error) in the funnel plot; asymmetry in a funnel plot indicates the presence of publication bias (23). Additionally, Egger's linear regression test (24) was adopted to assess the publication bias.

Where possible, trim and fill analyses were performed to verify the influence of publication bias on the overall pooled effect size. The trim and fill method was first proposed by Duval and
Tweedie (25); it aims to identify and correct asymmetry in funnel plots caused by publication bias. The trim and fill method not only evaluates the number of unpublished papers (missing data) in the meta-analysis, but also fills-in the missed data in the analysis to calibrate the pooled result.

\section{RESULTS}

\section{Description of Studies \\ Study Selection}

As presented in the PRISMA flow chart (Figure 1), a total of 96 articles were identified from four databases (PubMed: 31 , Web of Science: 37, Embase: 25, and Cochrane Library: 3), out of which 48 duplicates were removed. The remaining articles were viewed by title and abstract according to the inclusion criteria; 16 papers were eliminated for listed reasons and 32 papers were further checked by full-text. Finally, 11 articles [8-11, 15-17, and 2629] (incorporating 31 data reports) were involved in the metaanalysis and six more articles $(18,26-30)$ were involved in the systematic review.

\section{Characteristics of Included Studies}

The basic information of articles included in the meta-analysis is presented in Table 1. Of these articles, nine addressed the use of CPAE and five addressed CPEE. BG data were extracted from nine articles; TC and TG data were acquired from eight articles; HDL and LDL data were acquired from six articles; and OGTT data from four articles. Dosages ranged from 47 to $8,000 \mathrm{mg} / \mathrm{kg}$

\begin{tabular}{|c|c|c|c|c|c|c|}
\hline \multirow[t]{6}{*}{ CPAE } & Liu et al. (15) & $56.1 \%$ & $\begin{array}{c}34.5 \\
\pm 2.02(\mathrm{mg} / \mathrm{g})\end{array}$ & $0.55 \pm 0.003$ & $0.08 \pm 0.004$ & $0.12 \pm 0.003$ \\
\hline & & $53.1 \%$ & $\begin{array}{c}44.0 \pm \\
5.54(\mathrm{mg} / \mathrm{g})\end{array}$ & $1.99 \pm 0.021$ & $0.15 \pm 0.002$ & $1.53 \pm 0.026$ \\
\hline & & $58.0 \%$ & $\begin{array}{c}28.8 \pm \\
4.91(\mathrm{mg} / \mathrm{g})\end{array}$ & $0.52 \pm 0.003$ & $0.05 \pm 0.003$ & $0.06 \pm 0.003$ \\
\hline & & $53.6 \%$ & $\begin{array}{c}28.2 \pm \\
3.07(\mathrm{mg} / \mathrm{g})\end{array}$ & $0.60 \pm 0.010$ & $0.07 \pm 0.001$ & $0.13 \pm 0.009$ \\
\hline & Wang et al. (16) & $21.2 \%$ & $\begin{array}{c}4.13 \pm \\
0.072(\mathrm{mg} / \mathrm{g})\end{array}$ & $6.75 \pm 0.034$ & - & - \\
\hline & Xu et al. (10) & $1.5 \%$ & $68.11 \%$ & - & - & - \\
\hline \multirow[t]{6}{*}{ CPEE } & Wang et al. (16) & $18.64 \%$ & ND & $30.41 \pm 0.31$ & - & - \\
\hline & Liu et al. (15) & $48.7 \%$ & ND & $6.88 \pm 0.011$ & $5.69 \pm 0.012$ & $0.43 \pm 0.014$ \\
\hline & & $50.1 \%$ & ND & $4.70 \pm 0.002$ & $4.39 \pm 0.004$ & $1.57 \pm 0.154$ \\
\hline & & $49.1 \%$ & ND & $6.80 \pm 0.006$ & $8.15 \pm 0.018$ & $1.09 \pm 0.023$ \\
\hline & & $48.1 \%$ & ND & $5.90 \pm 0.008$ & $6.70 \pm 0.008$ & $0.71 \pm 0.034$ \\
\hline & & $48.5 \%$ & ND & $2.30 \pm 0.001$ & $7.70 \pm 0.001$ & $0.47 \pm 0.007$ \\
\hline
\end{tabular}

Content values are presented as mean $\pm S D$. CPAE, Cyclocarya paliurus aqueous extract; CPEE, Cyclocarya paliurus ethanol extract; ND, not detected. 
TABLE 3 | Mechanisms investigated in animal studies from 17 articles involved in the systematic review.

\begin{tabular}{|c|c|c|c|c|c|c|c|}
\hline Study & $\begin{array}{l}\text { Disease } \\
\text { model }\end{array}$ & $\begin{array}{l}\text { Pharmaceutical } \\
\text { composition }\end{array}$ & Animal & Gender & Pathways & $\begin{array}{l}\text { Protein/gene } \\
\text { detected }\end{array}$ & Major findings \\
\hline Jiang et al. (31) & Hyperlipidemia & $\begin{array}{l}\text { CPAE } \\
\text { CPEE }\end{array}$ & KM mice & $M$ & - & $\begin{array}{l}\text { HMG-CoA } \\
\text { reductase } \downarrow(E E), \\
\text { CYP7A1 } 1(E E), \\
A C A 2 \downarrow(E E)\end{array}$ & $\begin{array}{l}\text { BW } \downarrow \text {, food intake } \downarrow, T C \downarrow(E E), \\
\text { TG } \downarrow(E E), A S T \downarrow(E E), A L T \downarrow(E E), \\
\text { CRE } \downarrow(E E), \text { BUN } \downarrow(E E), \\
U A \downarrow(E E), \text { hepatic TC } \downarrow(E E), \\
\text { TG } \downarrow(E E) \text {, adipose size } \downarrow(E E), \\
\text { heart weight } \downarrow(A E) \text {, fat } \\
\text { weight } \downarrow(E E) \text {, hepatic bile } \\
\text { acid } \uparrow(E E), \text { fecal bile acid } \uparrow(E E)\end{array}$ \\
\hline Jiang et al. (7) & $\begin{array}{l}\text { Adipose } \\
\text { dysfunction } \\
\text { and insulin } \\
\text { resistance }\end{array}$ & CPEE & KM mice & $\mathrm{M}$ & $\begin{array}{l}\text { Insulin-Signaling: } \\
\text { IRS-1, AKT }\end{array}$ &  & $\begin{array}{l}\text { Hepatic and muscle } \\
\text { glycogen } \uparrow, A \cup C \text { of OGT } \downarrow\end{array}$ \\
\hline $\begin{array}{l}\text { Kurihara et al. } \\
\text { (32) }\end{array}$ & $\begin{array}{l}\text { Type } 2 \\
\text { diabetes }\end{array}$ & CPAE & $\mathrm{KK}-\mathrm{A}^{\mathrm{y}}$ mice & $\mathrm{M}$ & - & - & $\begin{array}{l}\text { AUC of OGTT } \downarrow, B G \downarrow, \\
\alpha \text {-glucosidase activity } \downarrow\end{array}$ \\
\hline $\begin{array}{l}\text { Kurihara et al. } \\
(30)\end{array}$ & $\begin{array}{l}\text { Post-prandial } \\
\text { hyperlipemia }\end{array}$ & CPAE & ICR mice & $M$ & - & - & TG $\downarrow$, pancreatic lipase $\downarrow$ \\
\hline Li et al. (29) & $\begin{array}{l}\text { Type } 2 \\
\text { diabetes }\end{array}$ & CPAE & KM mice & - & - & - & Percentage of glycemic $\downarrow$ \\
\hline Li et al. (33) & Diabetes & CPAE & SD Rats & $\mathrm{M}$ & $\begin{array}{l}\text { Cell proliferation, } \\
\text { cellular response to } \\
\text { glucose, insulin } \\
\text { stimulus }\end{array}$ & 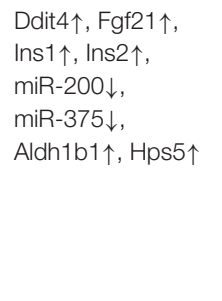 & $\begin{array}{l}\text { FBG } \downarrow, \text { AUC of OGTT } \downarrow, \mathrm{SI} \uparrow, \\
\text { islets area/total pancreatic } \\
\text { area } \uparrow \text {, thickness of glomerular } \\
\text { basement membrane } \downarrow, \mathrm{TC} \downarrow, \\
\text { TG } \downarrow, \mathrm{HDL}-\mathrm{L} / \mathrm{LDL}-\mathrm{c} \uparrow, \mathrm{AST} \downarrow, \\
\mathrm{ALT} \downarrow, \mathrm{CRE} \downarrow, \mathrm{BUN} \downarrow, \mathrm{FFA} \downarrow, \\
\mathrm{SOD} \uparrow, \mathrm{MDA} \downarrow, \text { glutathione } \uparrow, \\
\text { TNF- } \alpha \downarrow, \mathrm{IL}-6 \downarrow, \text { Ki67 positive } \\
\text { cells } \uparrow\end{array}$ \\
\hline Liu et al. (15) & $\begin{array}{l}\text { Type } 2 \\
\text { diabetes }\end{array}$ & $\begin{array}{l}\text { CPEE } \\
\text { CPAE }\end{array}$ & C57BL/6 & $M$ & - & - & $\begin{array}{l}\mathrm{FBG} \downarrow, \mathrm{BW} \uparrow, \mathrm{AUC} \text { of OGTT } \downarrow \text {, } \\
\mathrm{AUC} \text { of IT } \downarrow, \mathrm{TC} \downarrow, \mathrm{TG} \downarrow \\
\mathrm{HDL}-\mathrm{C} \uparrow(\mathrm{EE}), \mathrm{LDL}-\mathrm{C} \downarrow(\mathrm{EE}), \\
\mathrm{AST} \downarrow, \mathrm{ALT} \downarrow, \mathrm{TBI} \downarrow, \mathrm{CRE} \downarrow, \\
\mathrm{BUN} \downarrow\end{array}$ \\
\hline Ma et al. (11) & Hyperlipidemic & CPEE & $\mathrm{KM}$ mice & $M$ & $\begin{array}{l}\text { TNF- } \alpha / \text { p38MAPK } \\
\text { pathways: total- and } \\
\text { TRL-apoB48, } \\
\text { TNF- } \alpha, \text { p38MAPK }\end{array}$ & $\begin{array}{l}\text { Total- and } \\
\text { TRL-apoB48 } \downarrow \text {, } \\
\text { TNF- } \alpha \downarrow \\
\text { p-p38MAPK } \downarrow \\
\text { (intestinal tissue) }\end{array}$ & $\begin{array}{l}\mathrm{BW} \downarrow, \text { food intake } \downarrow, T C \downarrow, T G \downarrow \\
\text { HDL-C } \uparrow, \mathrm{LDL}-\mathrm{C} \downarrow, \mathrm{AST} \downarrow \\
\mathrm{ALT} \downarrow, \mathrm{CRE} \downarrow, \mathrm{BUN} \downarrow, \mathrm{MDA} \downarrow, \\
\mathrm{SOD} \uparrow, \mathrm{GSH}-\mathrm{Px} \uparrow\end{array}$ \\
\hline $\begin{array}{l}\text { Sheng et al. } \\
(17)\end{array}$ & $\begin{array}{l}\text { Type } 2 \\
\text { diabetes }\end{array}$ & CPAE & SD rats & $M$ & - & - & $\mathrm{FBG} \downarrow, \mathrm{AST} \downarrow, \mathrm{ALT} \downarrow, \mathrm{BUN} \downarrow$ \\
\hline $\begin{array}{l}\text { Wang et al. } \\
\text { (16) }\end{array}$ & $\begin{array}{l}\text { Type } 2 \\
\text { diabetes }\end{array}$ & $\begin{array}{l}\text { CPEE } \\
\text { CPAE }\end{array}$ & SD rats & $M$ & - & - & $\begin{array}{l}\text { Food intake } \downarrow, \mathrm{BG} \downarrow, \mathrm{FINS} \downarrow \\
\mathrm{GSH}-\mathrm{Px} \uparrow, \mathrm{FFA} \downarrow, \mathrm{TC} \downarrow, \mathrm{TG} \downarrow \\
\mathrm{LDL}-\mathrm{C} \downarrow, \mathrm{HDL}-\mathrm{C} \uparrow, \mathrm{SOD} \uparrow, \\
\mathrm{MDA} \downarrow, \mathrm{BUN} \downarrow, \mathrm{CRE} \downarrow(\mathrm{EE}), \\
\mathrm{GSP} \downarrow\end{array}$ \\
\hline $\begin{array}{l}\text { Wang et al. } \\
(34)\end{array}$ & $\begin{array}{l}\text { Diabetic } \\
\text { nephropathy }\end{array}$ & CPAE & $\begin{array}{l}\text { Wistar albino } \\
\text { rats }\end{array}$ & $M$ & - & - & $\begin{array}{l}\text { BG } \downarrow, \mathrm{SI} \uparrow, \mathrm{CRE} \downarrow, \mathrm{BUN} \downarrow, \\
\text { Upro } \downarrow, \mathrm{UG} \downarrow, \mathrm{UCr} \downarrow, \mathrm{CySC} \downarrow, \\
\alpha 1-\mathrm{MG} \text { levels } \downarrow, \mathrm{RI} \downarrow, \mathrm{IL}-6 \downarrow, \\
\mathrm{ET}-1 \downarrow, \mathrm{T}-\mathrm{AOC} \uparrow, \mathrm{SOD} \uparrow, \mathrm{MDA} \downarrow \text {, } \\
\text { GSH-Px } \downarrow, \mathrm{CAT} \uparrow, \text { renal AR } \\
\text { activity } \downarrow\end{array}$ \\
\hline Xiao et al. (9) & $\begin{array}{l}\text { Type } 2 \\
\text { diabetes }\end{array}$ & CPAE & $\begin{array}{l}\text { C57/BL6J } \\
\text { mice }\end{array}$ & $M$ & $\begin{array}{l}\text { MAPK pathway: } \\
\text { p38, ERK, JNK; Akt } \\
\text { pathway }\end{array}$ & 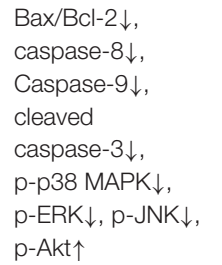 & $\begin{array}{l}\mathrm{BW} \uparrow, \mathrm{BG} \downarrow, \mathrm{SI} \uparrow, \mathrm{AUC} \text { of } \\
\mathrm{OGT} \downarrow, \mathrm{AST} \downarrow, \mathrm{ALT} \downarrow, \mathrm{ALP} \downarrow \\
\mathrm{TC} \downarrow, \mathrm{TG} \downarrow, \mathrm{HDL} \uparrow, \mathrm{LD} \downarrow \downarrow, \mathrm{CRE} \downarrow\end{array}$ \\
\hline
\end{tabular}


TABLE 3 | Continued

\begin{tabular}{|c|c|c|c|c|c|c|c|}
\hline Study & $\begin{array}{l}\text { Disease } \\
\text { model }\end{array}$ & $\begin{array}{l}\text { Pharmaceutical } \\
\text { composition }\end{array}$ & Animal & Gender & Pathways & $\begin{array}{l}\text { Protein/gene } \\
\text { detected }\end{array}$ & Major findings \\
\hline Xu et al. (10) & $\begin{array}{l}\text { Metabolic } \\
\text { syndrome }\end{array}$ & CPAE & SHR/cp rats & $\mathrm{M}$ & $\begin{array}{l}\text { Insulin-Signaling } \\
\text { Pathway: IRS1, } \\
\text { InsR, PI3K, AKT, } \\
\text { FoxO1, POMC, NPY }\end{array}$ & 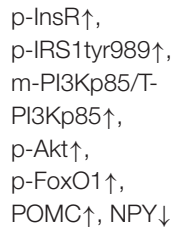 & $\begin{array}{l}\text { Food intake } \downarrow, \mathrm{BW} \downarrow, \\
\text { epididymal fat mass } \downarrow \text {, } \\
\text { abdominal fat mass } \downarrow, \mathrm{FBG} \downarrow, \\
\text { FINS } \downarrow, \mathrm{TC} \downarrow, \mathrm{TG} \downarrow, \mathrm{FFA} \downarrow, \\
\text { GOT } \downarrow, \mathrm{GPT} \downarrow, \mathrm{T}-\mathrm{GSH} \uparrow, \mathrm{SOD} \uparrow, \\
\text { MDA } \downarrow\end{array}$ \\
\hline Yao et al. (28) & $\begin{array}{l}\text { Hyperlipidemia } \\
\text { and obesity }\end{array}$ & CPEE & SD rats & $\mathrm{M}$ & - & - & $\begin{array}{l}\mathrm{BW} \downarrow, \mathrm{TC} \downarrow, \mathrm{TG} \downarrow, \mathrm{HDL}-\mathrm{C} \uparrow, \\
\mathrm{LDL}-\mathrm{C} \downarrow, \mathrm{AST} \downarrow, \mathrm{ALT} \downarrow, \mathrm{CRE} \downarrow \\
\mathrm{BUN} \downarrow, \mathrm{UA} \downarrow \text {, fat mass } \downarrow, \mathrm{FFA} \downarrow \text {, } \\
\text { serum leptin } \downarrow \text {, serum } \\
\text { adiponectin } \uparrow, \text { hepatic } \mathrm{TG} \downarrow \text {, } \\
\text { hepatic } \mathrm{TC} \downarrow \text {, fecal and hepatic } \\
\text { bile acid } \uparrow, \text { serum total } \\
\text { apoB48 } \downarrow\end{array}$ \\
\hline $\begin{array}{l}\text { Yoshitomi et al. } \\
(27)\end{array}$ & $\begin{array}{l}\text { Type } 2 \\
\text { diabetes }\end{array}$ & CPEE & ICR mice & $\mathrm{M}$ & - & $\begin{array}{l}\mathrm{p}-\mathrm{AS} 160 \uparrow \\
\mathrm{p}-\mathrm{AKT} \uparrow\end{array}$ & $\mathrm{BG} \downarrow, \mathrm{SI} \uparrow$ \\
\hline Zhai et al. (26) & $\begin{array}{l}\text { Type } 2 \\
\text { diabetes }\end{array}$ & CPEE & $\begin{array}{l}\text { C57/BL6J } \\
\text { mice }\end{array}$ & & - & - & $\begin{array}{l}\mathrm{BW} \uparrow, \mathrm{BG} \downarrow \text {, serum: TC } \downarrow, \mathrm{TG} \downarrow \\
\mathrm{LDL} \downarrow, \mathrm{HDL} \uparrow, \mathrm{AST} \downarrow, \mathrm{ALT} \downarrow \\
\text { hepatic } \mathrm{TC} \downarrow \text {, hepatic } \mathrm{TG} \downarrow\end{array}$ \\
\hline Zhao et al. (8) & $\begin{array}{l}\text { Type } 2 \\
\text { diabetes }\end{array}$ & CPEE & $\begin{array}{l}\text { C57/BL6J } \\
\text { mice }\end{array}$ & $\mathrm{F} / \mathrm{M}$ & - & - & $\begin{array}{l}\mathrm{BW} \uparrow, \mathrm{FBG} \downarrow, \mathrm{AUC} \text { of OGTT} \downarrow \\
\mathrm{SI} \downarrow, \mathrm{MDA} \downarrow, \mathrm{SOD} \uparrow, \mathrm{GSH}-\mathrm{Px} \uparrow\end{array}$ \\
\hline
\end{tabular}

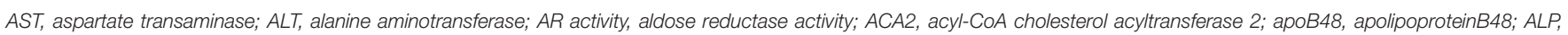

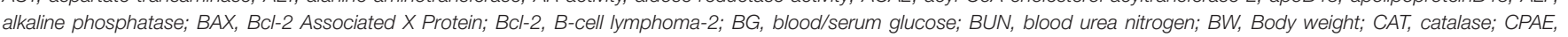

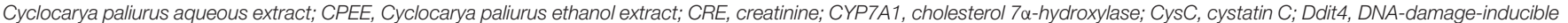

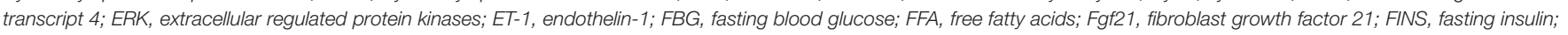

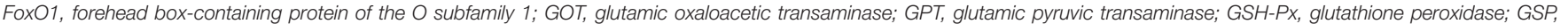

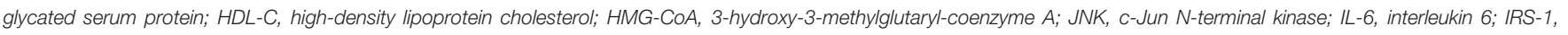

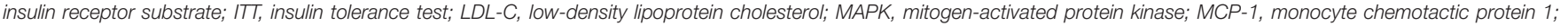



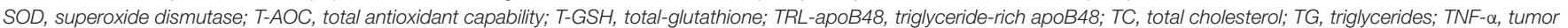
necrosis factor- $\alpha$; TBIL, total bilirubin; UA, uric acid; UCr, urine creatinine; UG, urine glucose; Upro, 24 h urine protein; $\alpha 1-M G, \alpha 1$ microglobulin. $\uparrow:$ up regulated.

$\downarrow$ : down regulated.

body weight, and the duration of included studies were all longer than 3 weeks. Animal models used in the studies could be divided into three types: (1) genetic models: KK-A $\mathrm{A}^{\mathrm{y}}$ mice, SHR/cp mice; (2) high-fat-diet (HFD)-induced models: KM mice with HFD; (3) HFD and chemical-induced models: streptozotocin (STZ) and alloxan are two chemicals widely used in developing diabetic models as both can cause damage to pancreatic $\beta$ cells (35).

\section{Risk of Bias}

A summary of the risk of bias for articles involved in metaanalyses is presented in Figure 2. Eleven articles showed unclear risk in most items using SYRCLE's tool for a lack of report of sequence generation, allocation concealment, and blinding. However, because the results of included outcomes were all objective parameters and difficult to be skewed, the blinding method for outcome assessment may not weigh as much in this meta-analysis when compared with other items.

\section{Chemicals in CPAE and CPEE}

Data of active compounds were acquired from articles involved in the systematic review (Table 2). CPAE mainly contains polysaccharides and CPEE is enriched with flavonoids and triterpenoids, while it contains almost no polysaccharides. In addition, polyphenols can be found in both extracts.
The major flavonoids identified from CP were: (1) quercetin-3-O-glucuronide, (2) quercetin-3-O-galactoside, (3) isoquercitrin, (4) kaempferol-3-O-glucuronide, (5) kaempferol-3-O-glucoside, (6) rhamnoside, (7) kaempferol3-O-rhamnoside, (8) kaempferol-3-O- $\alpha$-L-rhamnopyranoside, (9) kaempferol-3-O- $\beta$-D-glucuronide, and (10) quercetin (9, 11, 15, 27). Triterpenoids from CP mainly contained: (1) arjunolic acid, (2) cyclocaric acid B, (3) pterocaryoside B, (4) pterocaryoside A, (5) hederagenin, and (6) oleanolic acid $(11,15)$. Polyphenols from CP were mainly phenolic acids, which included: (1) 3-O-caffeoylquinic acid, (2) 4-O-caffeoylquinic acid, (3) 4,5-di-O-caffeoylquinic acid, (4) 1-caffeoylquinic acid, (5) chlorogenic acid, (6) 5-caffeoylquinic acid, and (7) cryptochlorogenic acid $(9,15,27)$. Though involved studies did not analyze the polysaccharides, other articles reported that the polysaccharide portion in CP is composed of: (1) rhamnose, (2) arabinose, (3) xylose, (4) mannose, (5) glucose, and (6) galactose $(19,36,37)$.

\section{Mechanisms Investigated in Previous Studies}

Molecular mechanisms from in vivo studies investigated in previous articles are summarized in Table 3. Only parameters showing statistical differences are presented. 


\section{Results From Meta-Analyses Effect of CP on Glucose Metabolism}

Both CPAE and CPEE showed definite hypoglycemic effects in animal models. CP extracts could lower BG level with an SMD of -3.20 (95\% CI: $-4.09,-2.31 ; P<0.001)$ and -5.73 (95\% CI: $-7.90,-3.56 ; P<0.001)$. Heterogeneity in the two subgroups was high $\left(\mathrm{I}^{2}=79,85 \%\right.$, respectively) (Supplementary Figure 1). Meta-regression showed that the effect size of $\mathrm{CP}$ on BG was related to the dosage used in treatment $(P<0.05)$, but not related to duration, model generation, or extract types. For the AUC of OGTT, the SMD results were -3.64 (95\% CI: -5.06 , $-2.23 ; P<0.001)$ for CPAE and -7.62 (95\% CI: $-11.01,-4.23)$ for CPEE (Supplementary Figure 2). Similarly, meta-regression showed the effect size was proportional to the dosage used in treatment. Furthermore, heterogeneity in subgroup analysis according to dosage $(<1,000,1,000-2,000,4,000,8,000 \mathrm{mg} / \mathrm{d})$ was partially reduced (Figures 3, 4). Sensitivity analyses were carried out by eliminating data from studies with a high risk of bias, however, they did not significantly reduce the heterogeneity between studies (Supplementary Table 2).

\section{Effect of CP on Lipid Metabolism}

The meta-analysis showed that CP has a beneficial effect on lipid profile. All outcomes could be improved by CPAE and CPEE. The overall pooled effect for TC, TG, HDL, LDL were -2.40 (95\% CI: $-2.89,-1.90 ; P<0.001),-1.90$ (95\% CI: -2.39 , $-1.42 ; P<0.001), 1.43$ (95\% CI: 1.04, 1.82; $P<0.001$ ), and -1.41 (95\% CI: $-1.81,-1.02 ; P<0.001)$, respectively. Forest plots for subgroup meta-analysis according to different extracts are shown in Supplementary Figures 3-6. In addition, Figure 5



FIGURE 3 | Forest plot for the effect of Cyclocarya paliurus (CP) on blood glucose levels in animal models. Subgroup analysis was performed according to doses. 


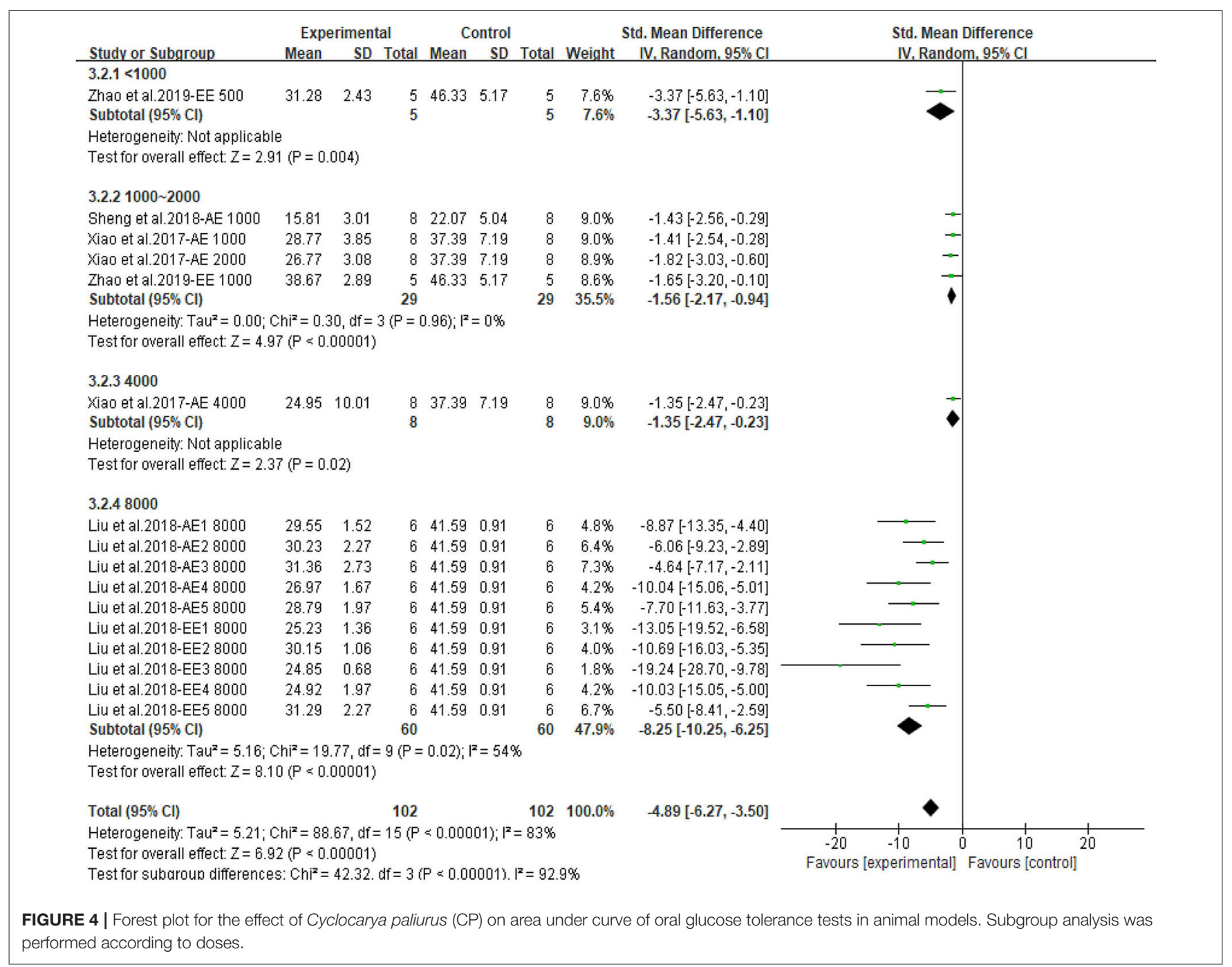

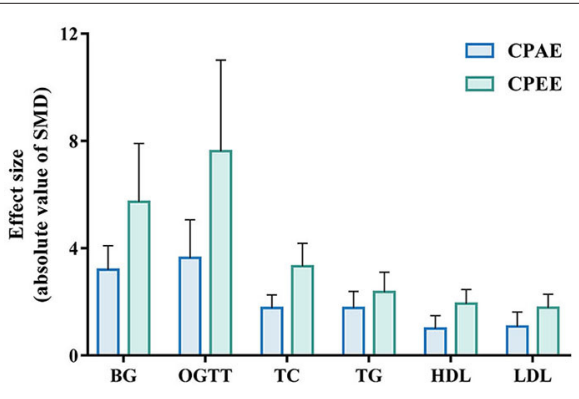

FIGURE 5 | Effect size of CPAE and CPEE on included parameters. CPAE, Cyclocarya paliurus aqueous extract; CPEE, Cyclocarya paliurus ethanol extract; BG, blood glucose level; OGTT, oral glucose tolerance test; TC, total cholesterol; TG, triglyceride; HDL, high-density lipoprotein; LDL, low-density lipoprotein.

compares the effect size of CPAE and CPEE on each parameter. Figures 6-9 are the forest plots generated according to different doses as subgroups.

\section{Publication Bias}

Asymmetry in the funnel plots indicates potential publication bias in the meta-analysis (Figure 10), the result was further confirmed by Egger's test with a $P$-value $<0.01$. Sample size in studies involved in this meta-analysis were rather small (no larger than 13), which could have caused an over-estimation of the overall effect size (38).

Only the result on HDL levels could be remedied by the trim and fill method (Supplementary Figure 7). Although the effect size on HDL level was estimated to be smaller, with an SMD = 0.936 (95\% CI: $0.498,1.375 ; P<0.001$ ), the beneficial effect was still steady. The trim and fill method failed to perform on other outcomes, possibly because of the existence of other biases or extremely positive and highly-weighted data (39).

\section{DISCUSSION}

Though CP has been used as a health-enhancing herbal tea for hundreds of years, the active components and underlying mechanisms still need to be uncovered. Various studies report 


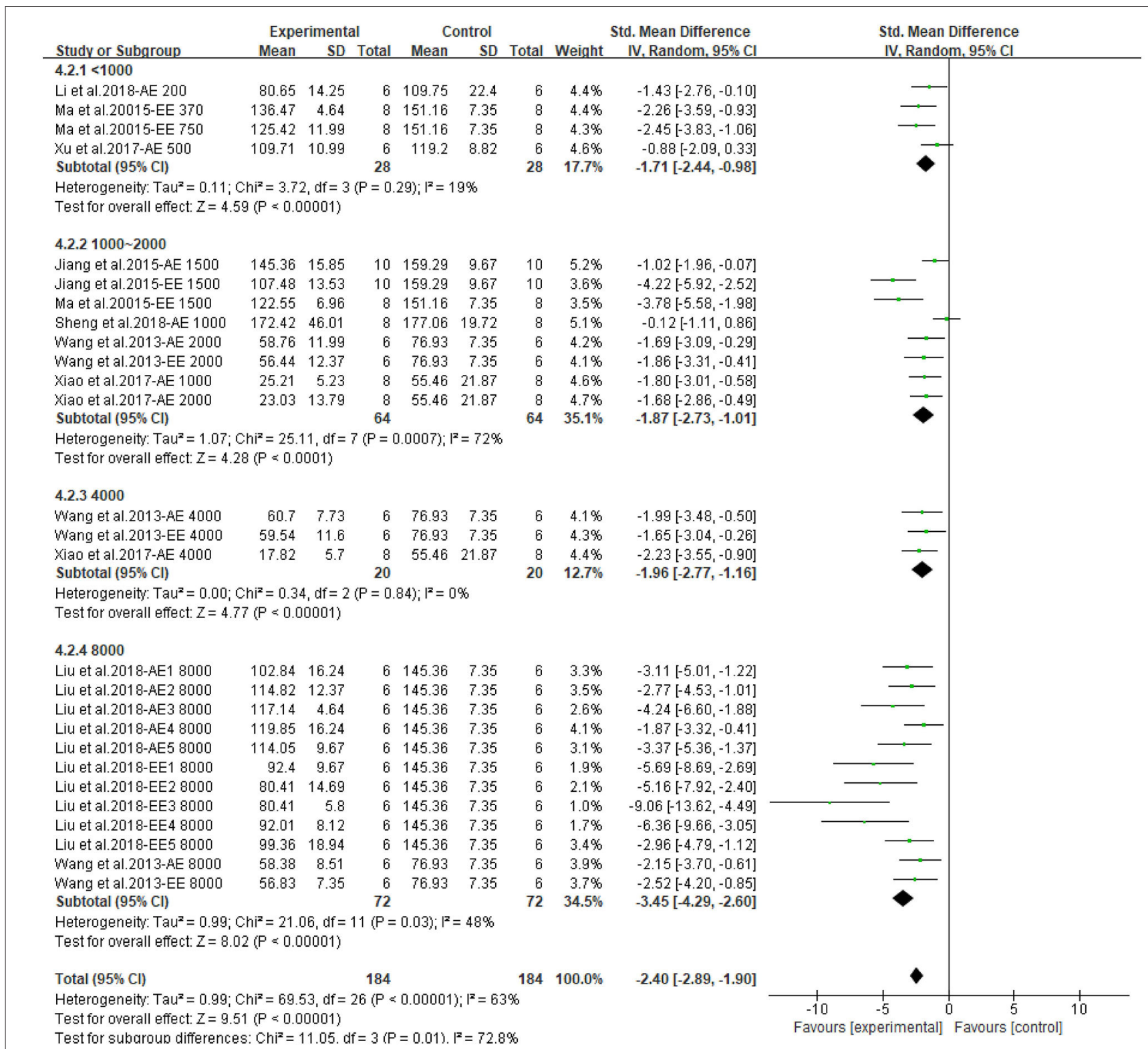

FIGURE 6 | Forest plot for the effect of Cyclocarya paliurus (CP) on total cholesterol levels in animal models. Subgroup analysis was performed according to doses.

CP's effect on regulating glucose homeostasis and improving lipid profiles, but no evidence-based research has been performed regarding its bioactivity for regulating glucolipid metabolism. This article aimed to collect current evidence to investigate the effect of $\mathrm{CP}$ on glucolipid metabolism through a metaanalysis. BG level and OGTT results were used to measure the hypoglycemic effect; TC, TG, HDL, and LDL were used to assess its effect on hyperlipidemia. The results showed that in animal studies, oral intake of $\mathrm{CP}$ extracts could effectively benefit glucolipid metabolism. BG level, as well as the AUC of OGTT were decreased; TC, TG, and LDL levels were decreased, while HDL level was increased. The effects of $\mathrm{CP}$ on glucolipid metabolism showed a dose-dependent manner: as the dosage rose, the effect size of the subgroup became larger.

Metabolic syndrome is a systematic metabolic dysfunction that involves insulin resistance, visceral adiposity, dyslipidemia, and hypertension (40). Insulin resistance is known to be the primary mechanism $(41,42)$, while other underlying mechanisms including oxidative stress (43), inflammation $(44,45)$, and endothelial dysfunction (46) are also discussed in literature. As is indicated by the criteria for clinical diagnosis of metabolic syndrome, abnormal carbohydrate and lipid metabolism are highly associated with this syndrome (5).

After its secretion by the pancreatic $\beta$ cells, insulin binds with insulin receptors on target cells to perform its biofunctions. 


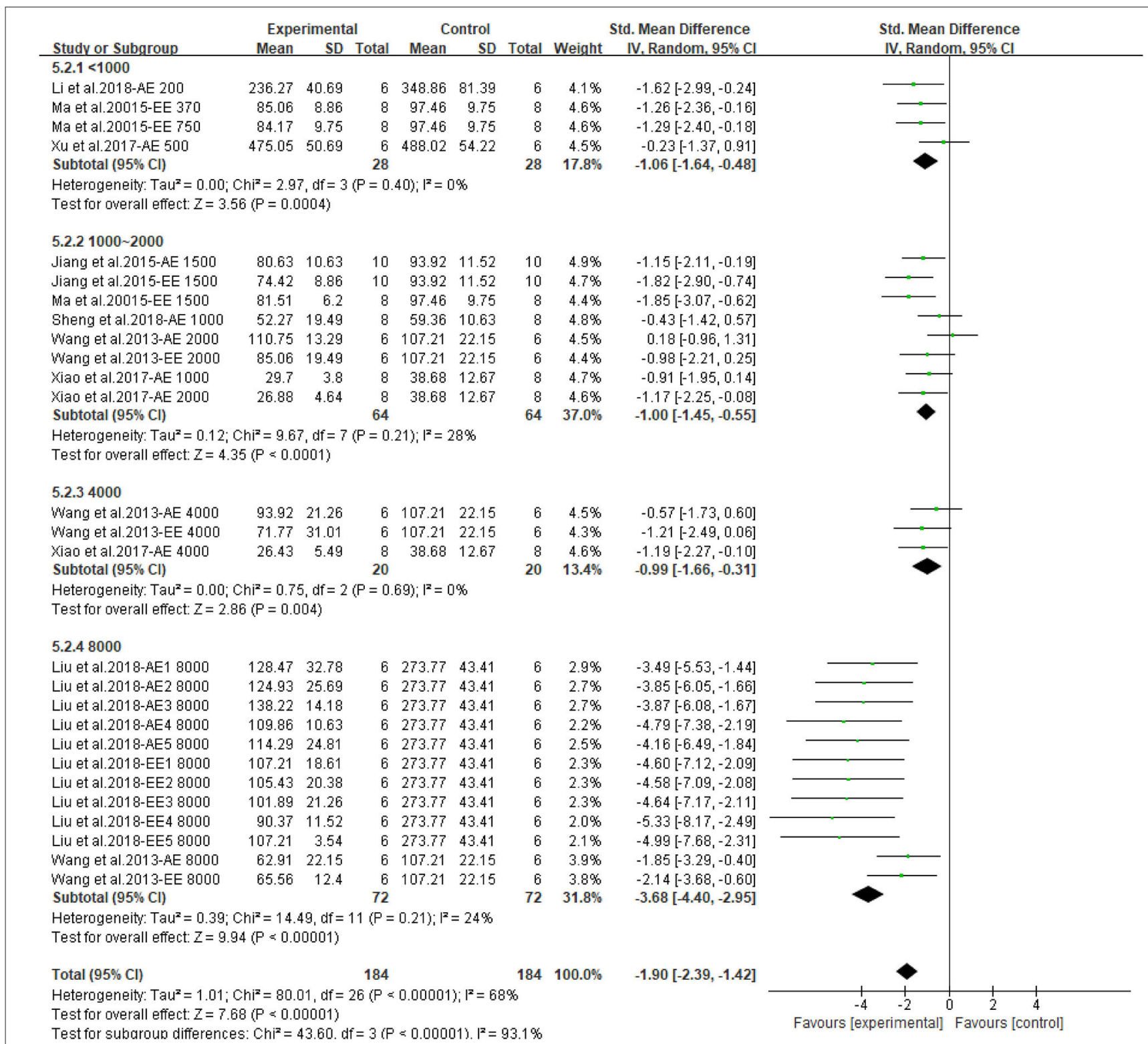

FIGURE 7 | Forest plot for the effect of Cyclocarya paliurus (CP) on triglyceride levels in animal models. Subgroup analysis was performed according to doses.

Obstruction to insulin signaling pathways could lead to insulin resistance (47), causing hyperinsulinemia, which encourages other pathological changes associated with metabolic syndrome, including dyslipidemia, hypertension, as well as the progress of type 2 diabetes (42). CP was shown to decrease food intake in mice $(10,11,31)$, this effect is probably due to its function in improving insulin signaling in the hypothalamus, and thus regulating the appetite regulating neuron neuropeptide $\mathrm{Y}$ (NPY) and proopiomelanocortin (POMC), leading to inhibition of food intake (10). In muscle tissues, CP also increases the activation of insulin receptor substrate-1(IRS-1), which further activates downstream proteins, such as phosphoinositide 3-kinases (PI3K), protein kinase B (Akt), and glucose transporter type 4 (GLUT4), and enhances glucose disposal in muscles (7).

It has been suggested that insulin resistance and hyperinsulinemia would then cause increasing pancreatic $\beta$ cell apoptosis, leading to a decrease in $\beta$ cell mass, as a loss of $\beta$ cell mass due to apoptotic death is observed in patients with type 2 diabetes $(48,49)$. In animal studies, CP was shown to decrease pancreatic $\beta$ cell apoptosis, possibly due to the regulation of mitogen-activated protein kinase (MAPK) pathways and Akt pathways (9). Furthermore, restoration of $\beta$ cell mass and function were found in histological observations, moreover, this $\beta$-cell-protecting effect was also confirmed by in vitro studies in 


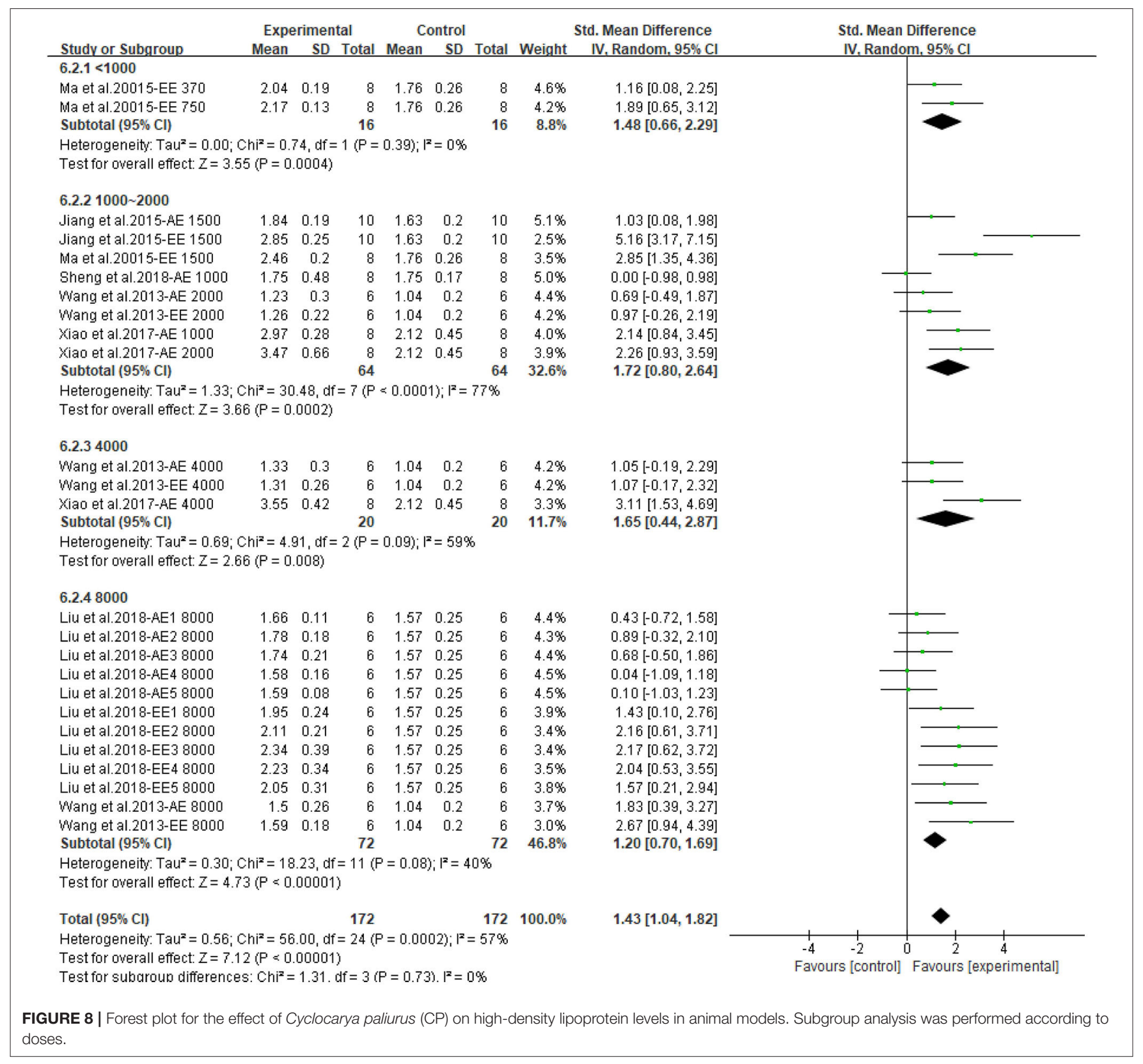

NIT- 1 cells $(8,9)$, which are a pancreatic $\beta$ cell line established from mice (50).

Oxidative stress and inflammation are also linked to metabolic syndrome. The expression of markers for inflammation and oxidative stress were found to be much higher in patients with metabolic syndrome than those without it (51). Oxidative stress is characterized by the production and degradation of reactive oxygen species (ROS) and over-production of oxidative free radicals (52), which occurs along with inflammation when adipocytes fail to store excessive energy (53). Inflammatory cytokines like tumor necrosis factor- $\alpha$ (TNF- $\alpha$ ) down-regulate the activation of insulin receptors (54), and eventually lead to insulin resistance, causing hypertriglyceridemia, low HDL levels and high LDL levels (55). In many current animal studies, CP administration successfully decreases the expression of TNF$\alpha$, interleukin-6 (IL-6), malondialdehyde (MDA), and increases superoxide dismutase (SOD) and glutathione peroxidase (GSH$\mathrm{Px}$ ) levels, showing its capacity for controlling inflammation and oxidative stress $(8,10,11,16)$.

Other underlying mechanisms have also been investigated in animal models. Apolipoprotein B48 (apoB48) is a protein that transports exogenous fat into the circulation and other body parts (56). ApoB48 promotes the absorption of exogenous lipid in the intestine, and overexpression of apoB48 may result in hyperlipidemia (57). CP significantly lowered serum apoB48 content in hyperlipidemic models, probably because of modulation in the MAPK pathway $(11,28)$. Leptin and adiponectin are two major adipocytokines, both play important 


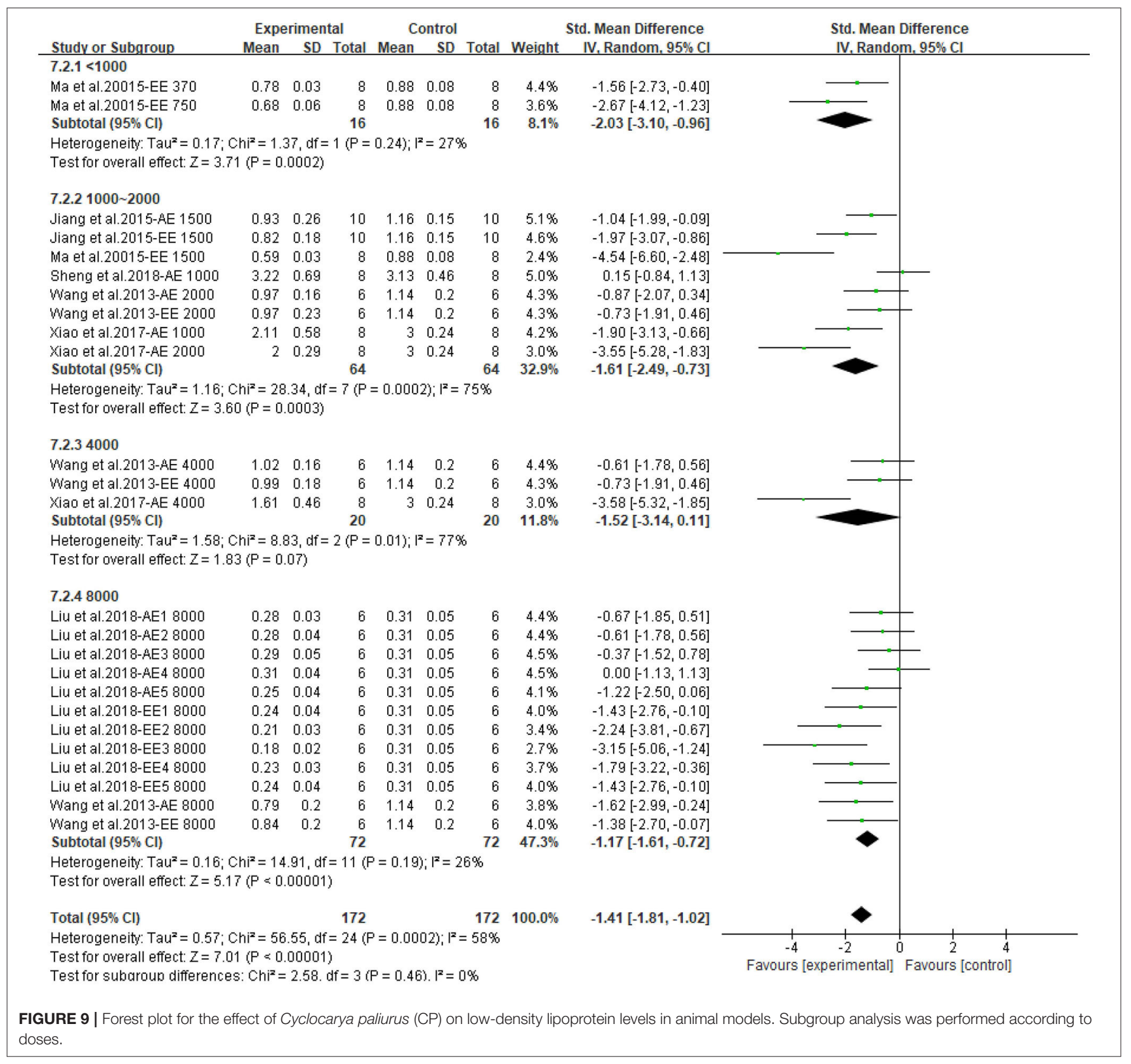

roles in metabolic homeostasis. It has been pointed out that the ratio of leptin to adiponectin is a better indicator of insulin resistance and atherosclerosis than each alone (58). The research of Yao et al. found that $\mathrm{CP}$ significantly reduced the leptin to adiponectin ratio, indicating that $\mathrm{CP}$ protects against obesity through regulating the secretion of adipocytokines (28). CP was also demonstrated to have the ability to inhibit $\alpha$-glucosidase activity (30). Furthermore, a transcriptome study revealed more possible mechanisms: $\mathrm{CP}$ improved $\beta$-cell survival and insulin secretion, and regulated the miR-200/375-Aldh1b1/Hps5-Hes1 co-regulatory network to restore lipid metabolism and exert its anti-oxidant ability (33).

The mechanisms investigated in articles involved in this systematic review are summarized and presented in Figure 11.
From the hypothalamus to the intestine, CP targets multiple organs and tissues, and systematically regulates glucolipid metabolism and benefits metabolic syndrome in vivo.

It is worth noting that in this meta-analysis, CPEE had a greater effect on every calculated parameter than CPAE (Figure 5). In addition, in some studies involving both extracts, CPEE also seemed to be more effective for improving phenotypes and regulating proteins $(15,31)$. However, this effect might have been caused by an inherent bias of individual studies or across studies. Furthermore, publication bias and selective reporting may also have affected the cumulative evidence. Flavonoids and triterpenoids are more likely to be the effective compounds in CPEE, and further studies performed on these phytochemicals have shown positive results $(13,18,59-61)$. Meanwhile, as the 
main components found in CPAE, polysaccharides have been previously investigated. For example, polysaccharides in $\mathrm{CP}$ could ameliorate hyperlipidemia via regulating DNA methylation levels and regulating lipid metabolism-related enzymes in the liver $(36,37)$. It has been pointed out that the bioavailability of flavonoids is low, as a large proportion of them will remain in the digestive system after oral intake $(62,63)$. However, Cheng's

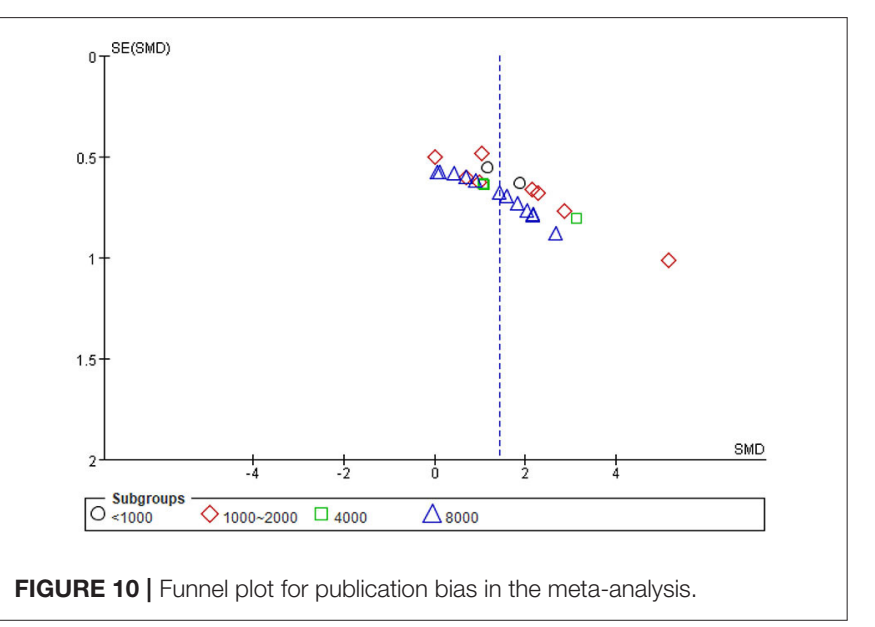

study showed that CP flavonoids can modulate gut microbiota to regulate certain metabolic processes, to the benefit of murine obesity models (60).

In addition, a toxicity study reported that, even when given at the highest dosage (10 g/ kg in Kunming mice and $20 \mathrm{~g} / \mathrm{kg}$ in SD rats), CPAE did not result in any mutagenicity, sperm deformity, or teratogenicity in rodents, even when the dose was 150-fold of the recommended human dose (64). This suggests that CP is safe to be used as an additive ingredient in daily nutritional supplements, with $6 \mathrm{~g}$ of crude extract proposed to be the effective daily dosage for a $50 \mathrm{~kg}$ human (28). No reports regarding any side effects of the oral intake of CP were found, and this point is worth further investigation.

This article has some limitations. Firstly, according to the assessment of risk of bias, the involved studies showed an unclear risk in most items, which is common in animal studies (65). Ambiguous descriptions of experimental methods may have led to skewed outcomes and eventually affected the pooled results of the meta-analysis. Moreover, publication bias may have enlarged the effect size of CP. However, for the inevitable heterogeneity in preclinical studies, the results of this meta-analysis should be interpreted as a guide to the direction of outcomes instead of viewed as precise estimates of the pooled effect size. No randomcontrolled clinical trials were found in databases, and additional

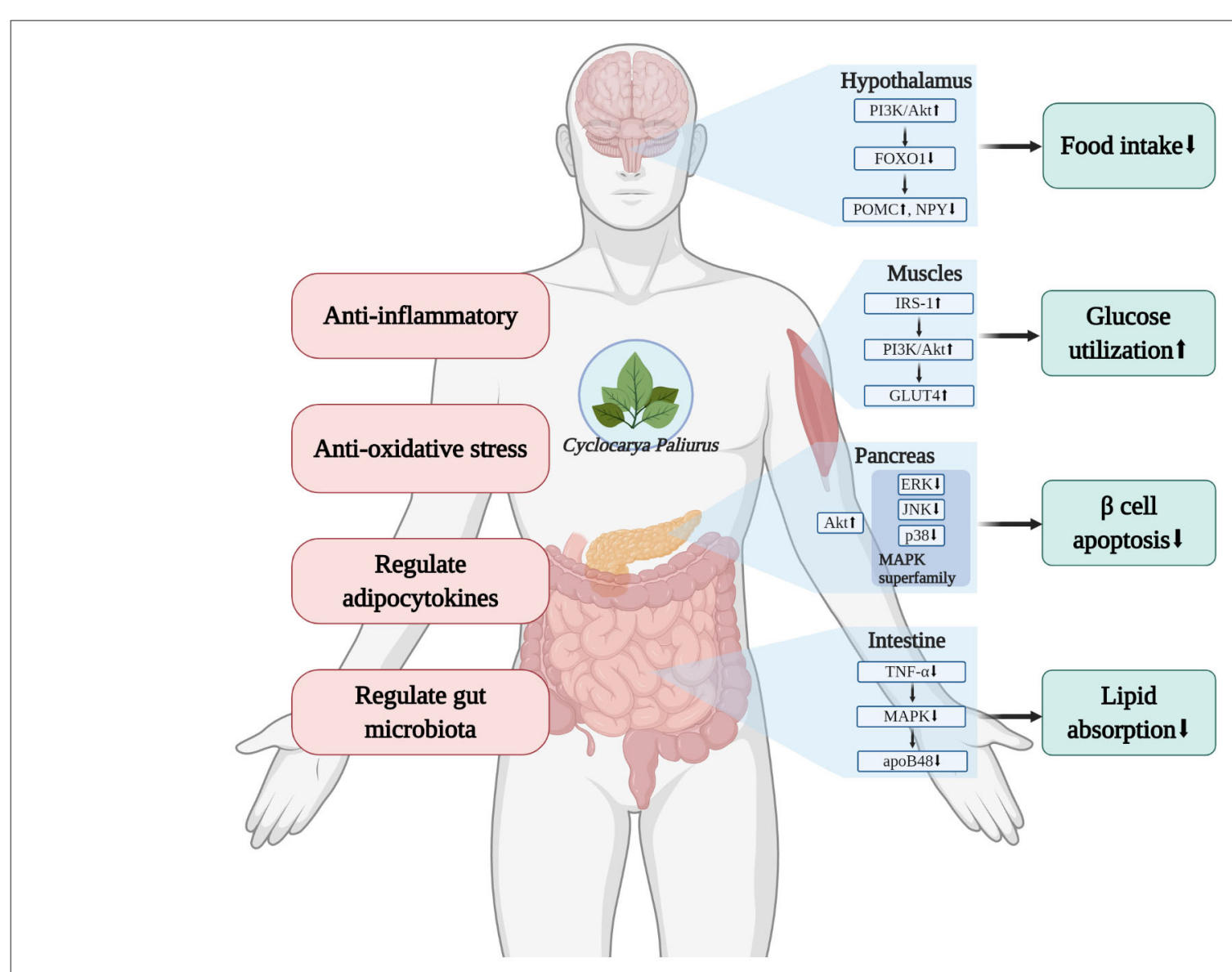

FIGURE 11 | Diagram of the mechanisms investigated for the effect of Cyclocarya paliurus (CP) on glucolipid metabolism (created with BioRender.com). 
studies are still needed to identify more potential therapeutic targets, and form a more comprehensive understanding of CP.

\section{CONCLUSION}

According to this meta-analysis, oral intake of CP showed absolute ameliorative effects on glucolipid metabolism in animal models. In addition, the studied molecular pathways in previous literature were systematically reviewed. CP ameliorates metabolic syndrome probably through enhancing insulin sensitivity, decreasing pancreatic $\beta$ cell apoptosis, inhibiting lipid absorption, regulating adipocytokines and gut microbiota, and combatting inflammation and oxidative stress.

As a daily dietary supplement, CP showed promising potential for dealing with metabolic syndrome by influencing multiple related mechanisms. In addition, future research should focus on the composition of CPEE.

\section{DATA AVAILABILITY STATEMENT}

The original contributions generated for the study are included in the article/Supplementary Material, further inquiries can be directed to the corresponding author/s.

\section{REFERENCES}

1. Eckel RH, Grundy SM, Zimmet PZ. The metabolic syndrome. Lancet. (2005) 365:1415-28. doi: 10.1016/s0140-6736(05)66378-7

2. Grundy SM. Metabolic syndrome: connecting and reconciling cardiovascular and diabetes worlds. J Am Coll Cardiol. (2006) 47:1093-100. doi: 10.1016/j.jacc.2005.11.046

3. Saklayen MG. The global epidemic of the metabolic syndrome. Curr Hypertens Rep. (2018) 20:12. doi: 10.1007/s11906-0180812-z

4. Samson SL, Garber AJ. Metabolic syndrome. Endocrinol Metab Clin North Am. (2014) 43:1-23. doi: 10.1016/j.ecl.2013.09.009

5. Alberti KG, Eckel RH, Grundy SM, Zimmet PZ, Cleeman JI, Donato $\mathrm{KA}$, et al. Harmonizing the metabolic syndrome: a joint interim statement of the International Diabetes Federation Task Force on Epidemiology and Prevention; National Heart, Lung, and Blood Institute; American Heart Association; World Heart Federation; International Atherosclerosis Society; and International Association for the Study of Obesity. Circulation. (2009) 120:1640-5. doi: 10.1161/circulationaha.109. 192644

6. Bergman RN, Kim SP, Catalano KJ, Hsu IR, Chiu JD, Kabir M, et al. Why visceral fat is bad: mechanisms of the metabolic syndrome. Obesity. (2006) 14:16s-9s. doi: 10.1038/oby.2006.277

7. Jiang C, Yao N, Wang Q, Zhang J, Sun Y, Xiao N, et al. Cyclocarya paliurus extract modulates adipokine expression and improves insulin sensitivity by inhibition of inflammation in mice. J Ethnopharmacol. (2014) s153:344-51. doi: 10.1016/j.jep.2014.02.003

8. Zhao L, Wang X, Li J, Tan X, Fan L, Zhang Z, et al. Effect of Cyclocarya paliurus on hypoglycemic effect in type 2 diabetic mice. Med Sci Monit. (2019) 25:2976-83. doi: 10.12659/MSM.913368

9. Xiao HT, Wen B, Ning ZW, Zhai LX, Liao CH, Lin CY, et al. Cyclocarya paliurus tea leaves enhances pancreatic $\beta$ cell preservation through inhibition of apoptosis. Sci Rep. (2017) 7:9155. doi: 10.1038/s41598-017-09641-Z

10. Xu G, Yoshitomi H, Sun W, Guo X, Wu L, Guo X, et al. Cyclocarya paliurus (Batal.) Ijinskaja aqueous extract (CPAE) ameliorates obesity by improving insulin signaling in the hypothalamus of a metabolic syndrome rat model. J Evid Based Complement Altern Med. (2017) 2017:1-9. doi: $10.1155 / 2017 / 4602153$

\section{AUTHOR CONTRIBUTIONS}

YW, WL, TL, and LW designed this research. WL, YH, and SQ participated in the literature search and selection. XG and MW worked on data extraction. YW and WL organized the data and performed the analysis. YW wrote the original manuscript. All authors contributed to the article and approved the submitted version.

\section{FUNDING}

This work was supported by the Creation and Talent Introduction Base of Prevention and Treatment of Diabetes and Its Complications with Traditional Chinese Medicine (Grant No. B20055).

\section{SUPPLEMENTARY MATERIAL}

The Supplementary Material for this article can be found online at: https://www.frontiersin.org/articles/10.3389/fnut.2020. 605605/full\#supplementary-material

11. Ma Y, Jiang C, Yao N, Li Y, Wang Q, Fang S, et al. Antihyperlipidemic effect of Cyclocarya paliurus (Batal.) Iljinskaja extract and inhibition of apolipoprotein B48 overproduction in hyperlipidemic mice. J Ethnopharmacol. (2015) 166:286-96. doi: 10.1016/j.jep.2015. 03.030

12. Xie JH, Xie MY, Nie SP, Shen MY, Wang YX, Li C. Isolation, chemical composition and antioxidant activities of a water-soluble polysaccharide from Cyclocarya paliurus (Batal.) Iljinskaja. Food Chem. (2010) 119:1626-32. doi: 10.1016/j.foodchem.2009.09.055

13. Wu Z, Gao T, Zhong R, Lin Z, Jiang C, Ouyang S, et al. Antihyperlipidaemic effect of triterpenic acid-enriched fraction from Cyclocarya paliurus leaves in hyperlipidaemic rats. Pharm Biol. (2017) 55:712-21. doi: 10.1080/13880209.2016.1267231

14. Zhang $\mathrm{XX}$, Jiang $\mathrm{CH}$, Liu $\mathrm{Y}$, Lou DX, Huang $\mathrm{YP}$, Gao $\mathrm{M}$, et al. Cyclocarya paliurus triterpenic acids fraction attenuates kidney injury via AMPK-mTOR-regulated autophagy pathway in diabetic rats. Phytomedicine. (2019) 64:153060. doi: 10.1016/j.phymed.2019. 153060

15. Liu Y, Cao Y, Fang S, Wang T, Yin Z, Shang X, et al. Antidiabetic effect of Cyclocarya paliurus leaves depends on the contents of antihyperglycemic flavonoids and antihyperlipidemic triterpenoids. Molecules. (2018) 23:1042. doi: 10.3390/molecules23051042

16. Wang QQ, Jiang $\mathrm{CH}$, Fang SZ, Wang JH, Ji Y, Shang XL, et al. Antihyperglycemic, antihyperlipidemic and antioxidant effects of ethanol and aqueous extracts of Cyclocarya paliurus leaves in type 2 diabetic rats. $J$ Ethnopharmacol. (2013) 150:1119-27. doi: 10.1016/j.jep.2013.10.040

17. Sheng X, Zhao M, Jiang C, Zhang J, Yin Z. Hypoglycemic effect of medicinal formula composed of Cyclocarya paliurus and mulberry leaves. J China Pharm Univ. (2018) 49:463-9. doi: 10.11665/j.issn.1000-5048.20 180412

18. Jiang C, Wang Y, Jin Q, Zhang D, Gao M, Yao N, et al. Cyclocarya paliurus triterpenoids improve diabetes-induced hepatic inflammation via the rho-kinase-dependent pathway. Front Pharmacol. (2019) 10:811. doi: 10.3389/fphar.2019.00811

19. Yang Z, Wang J, Li J, Xiong L, Chen H, Liu X, et al. Antihyperlipidemic and hepatoprotective activities of polysaccharide fraction from Cyclocarya paliurus in high-fat emulsion-induced hyperlipidaemic mice. Carbohydr Polym. (2018) 183:11-20. doi: 10.1016/j.carbpol.2017.11.033 
20. Moher D, Liberati A, Tetzlaff J, Altman DG. Preferred reporting items for systematic reviews and meta-analyses: the PRISMA statement. PLoS Med. (2009) 6:e1000097. doi: 10.1371/journal.pmed.10 00097

21. Hooijmans CR, Rovers MM, de Vries RB, Leenaars M, RitskesHoitinga M, Langendam MW. SYRCLE's risk of bias tool for animal studies. BMC Med Res Methodol. (2014) 14:43. doi: 10.1186/1471-22 88-14-43

22. Higgins JP, Thompson SG, Deeks JJ, Altman DG. Measuring inconsistency in meta-analyses. BMJ. (2003) 327:557-60. doi: $10.1136 / \mathrm{bmj} .327 .74$ 14.557

23. Debray TPA, Moons KGM, Riley RD. Detecting small-study effects and funnel plot asymmetry in meta-analysis of survival data: a comparison of new and existing tests. Res Synth Methods. (2018) 9:41-50. doi: 10.1002/ jrsm.1266

24. Egger M, Davey Smith G, Schneider M, Minder C. Bias in metaanalysis detected by a simple, graphical test. BMJ. (1997) 315:629-34. doi: $10.1136 / \mathrm{bmj} .315 .7109 .629$

25. Duval S, Tweedie R. Trim and fill: a simple funnel-plot-based method of testing and adjusting for publication bias in meta-analysis. Biometrics. (2000) 56:455-63. doi: 10.1111/j.0006-341x.2000.00455.x

26. Zhai L, Ning ZW, Huang T, Wen B, Liao CH, Lin CY, et al. Cyclocarya paliurus leaves tea improves dyslipidemia in diabetic mice: a lipidomicsbased network pharmacology study. Front Pharmacol. (2018) 9:973. doi: 10.3389/fphar.2018.00973

27. Yoshitomi H, Tsuru R, Li L, Zhou J, Kudo M, Liu T, et al. Cyclocarya paliurus extract activates insulin signaling via Sirtuin1 in C2C12 myotubes and decreases blood glucose level in mice with impaired insulin secretion. PLoS ONE. (2017) 12:e0183988. doi: 10.1371/journal.pone.0183988

28. Yao X, Lin Z, Jiang C, Gao M, Wang Q, Yao N, et al. Cyclocarya paliurus prevents high fat diet induced hyperlipidemia and obesity in SpragueDawley rats. Can J Physiol Pharmacol. (2015) 93:677-86. doi: 10.1139/cjpp2014-0477

29. Li S, Li J, Guan XL, Li J, Deng SP, Li LQ, et al. Hypoglycemic effects and constituents of the barks of Cyclocarya paliurus and their inhibiting activities to glucosidase and glycogen phosphorylase. Fitoterapia. (2011) 82:1081-5. doi: 10.1016/j.fitote.2011.07.002

30. Kurihara H, Asami S, Shibata H, Fukami H, Tanaka T. Hypolipemic effect of Cyclocarya paliurus (Batal) Iljinskaja in lipid-loaded mice. Biol Pharm Bull. (2003) 26:383-5. doi: 10.1248/bpb.26.383

31. Jiang C, Wang Q, Wei Y, Yao N, Wu Z, Ma Y, et al. Cholesterol-lowering effects and potential mechanisms of different polar extracts from Cyclocarya paliurus leaves in hyperlipidemic mice. J Ethnopharmacol. (2015) 176:17-26. doi: $10.1016 /$ j.jep.2015.10.006

32. Kurihara H, Fukami H, Kusumoto A, Toyoda Y, Shibata H, Matsui Y, et al. Hypoglycemic action of Cyclocarya paliurus (Batal.) Iljinskaja in normal and diabetic mice. Biosci Biotechnol Biochem. (2003) 67:877-80. doi: $10.1271 / \mathrm{bbb} .67 .877$

33. Li J, Luo $\mathrm{M}, \mathrm{Hu} \mathrm{MH}$, Guo $\mathrm{AY}$, Yang $\mathrm{XL}$, Zhang $\mathrm{Q}$, et al. Investigating the molecular mechanism of aqueous extract of Cyclocarya paliurus on ameliorating diabetes by transcriptome profiling. Front Pharmacol. (2018) 9:912. doi: 10.3389/fphar.2018.00912

34. Wang XH, Li WZ, Kong D. Cyclocarya paliurus extract alleviates diabetic nephropathy by inhibiting oxidative stress and aldose reductase. Renal Failure. (2016) 38:678-85. doi: 10.3109/0886022x.2016.1155394

35. Lenzen S. The mechanisms of alloxan- and streptozotocin-induced diabetes. Diabetologia. (2008) 51:216-26. doi: 10.1007/s00125-007-0886-7

36. Hu WB, Zhao J, Chen H, Xiong L, Wang WJ. Polysaccharides from Cyclocarya paliurus: Chemical composition and lipid-lowering effect on rats challenged with high-fat diet. J Funct Foods. (2017) 36:262-73. doi: 10.1016/j.jff.2017.07.020

37. Yang ZW, Ouyang KH, Zhao J, Chen H, Xiong L, Wang WJ. Structural characterization and hypolipidemic effect of Cyclocarya paliurus polysaccharide in rat. Int J Biol Macromol. (2016) 91:1073-80. doi: 10.1016/j.ijbiomac.2016.06.063

38. Park HS, Cho JH, Kim KW, Chung WS, Song MY. Effects of Panax ginseng on obesity in animal models: a systematic review and meta-analysis. J Evid Based Complement Altern Med. (2018) 2018:2719794. doi: 10.1155/2018/2719794
39. Thomas JS. Nonparametric trim and fill analysis of publication bias in metaanalysis. Stata Tech Bull. (2000) 10:8-14.

40. Huang PL. A comprehensive definition for metabolic syndrome. Dis Models Mech. (2009) 2:231-7. doi: $10.1242 / \mathrm{dmm} .001180$

41. Reaven GM. Banting lecture 1988. Role of insulin resistance in human disease. Diabetes. (1988) 37:1595-607. doi: 10.2337/diab.37.12.1595

42. Haffner SM, Valdez RA, Hazuda HP, Mitchell BD, Morales PA, Stern MP. Prospective analysis of the insulin-resistance syndrome (syndrome X). Diabetes. (1992) 41:715-22. doi: 10.2337/diab.41.6.715

43. Vona R, Gambardella L, Cittadini C, Straface E, Pietraforte D. Biomarkers of oxidative stress in metabolic syndrome and associated diseases. Oxid Med Cell Longev. (2019) 2019:8267234. doi: 10.1155/2019/8267234

44. Esser N, Legrand-Poels S, Piette J, Scheen AJ, Paquot N. Inflammation as a link between obesity, metabolic syndrome and type 2 diabetes. Diabetes Res Clin Pract. (2014) 105:141-50. doi: 10.1016/j.diabres.2014.04.006

45. Hotamisligil GS. Inflammation and metabolic disorders. Nature. (2006) 444:860-7. doi: 10.1038/nature05485

46. Shayo SC, Kawade S, Ogiso K, Yoshihiko N. Strategies to ameliorate endothelial dysfunction associated with metabolic syndrome, where are we? Diabetes Metab Syndr. (2019) 13:2164-9. doi: 10.1016/j.dsx.2019.05.005

47. Czech MP. Insulin action and resistance in obesity and type 2 diabetes. Nat Med. (2017) 23:804-14. doi: 10.1038/nm.4350

48. Butler AE, Janson J, Bonner-Weir S, Ritzel R, Rizza RA, Butler PC. Betacell deficit and increased beta-cell apoptosis in humans with type 2 diabetes. Diabetes. (2003) 52:102-10. doi: 10.2337/diabetes.52.1.102

49. Hanley NA, Hanley KP, Miettinen PJ, Otonkoski T. Weighing up beta-cell mass in mice and humans: self-renewal, progenitors or stem cells? Mol Cell Endocrinol. (2008) 288:79-85. doi: 10.1016/j.mce.2008.03.001

50. Hamaguchi K, Gaskins HR, Leiter EH. NIT-1, a pancreatic beta-cell line established from a transgenic NOD/Lt mouse. Diabetes. (1991) 40:842-9. doi: $10.2337 /$ diab.40.7.842

51. Van Guilder GP, Hoetzer GL, Greiner JJ, Stauffer BL, Desouza CA. Influence of metabolic syndrome on biomarkers of oxidative stress and inflammation in obese adults. Obesity. (2006) 14:2127-31. doi: 10.1038/oby.2006.248

52. Furukawa S, Fujita T, Shimabukuro M, Iwaki M, Yamada Y, Nakajima Y, et al. Increased oxidative stress in obesity and its impact on metabolic syndrome. $J$ Clin Invest. (2004) 114:1752-61. doi: 10.1172/jci21625

53. Klöting $N$, Blüher $M$. Adipocyte dysfunction, inflammation and metabolic syndrome. Rev Endocr Metab Disord. (2014) 15:277-87. doi: 10.1007/s11154-014-9301-0

54. Andrade-Oliveira V, Câmara NO, Moraes-Vieira PM. Adipokines as drug targets in diabetes and underlying disturbances. J Diabetes Res. (2015) 2015:681612. doi: $10.1155 / 2015 / 681612$

55. Grundy SM. Hypertriglyceridemia, atherogenic dyslipidemia, and the metabolic syndrome. Am J Cardiol. (1998) 81:18b-25b. doi: 10.1016/s0002-9149(98)00033-2

56. Klop B, Elte JW, Cabezas MC. Dyslipidemia in obesity: mechanisms and potential targets. Nutrients. (2013) 5:1218-40. doi: 10.3390/nu5041218

57. Ginsberg HN. Lipoprotein physiology. Endocrinol Metab Clin North Am. (1998) 27:503-19. doi: 10.1016/s0889-8529(05)70023-2

58. Satoh N, Naruse M, Usui T, Tagami T, Suganami T, Yamada K, et al. Leptinto-adiponectin ratio as a potential atherogenic index in obese type 2 diabetic patients. Diabetes Care. (2004) 27:2488-90. doi: 10.2337/diacare.27.10.2488

59. Xie J, Wang W, Dong C, Huang L, Wang H, Li C, et al. Protective effect of flavonoids from Cyclocarya paliurus leaves against carbon tetrachlorideinduced acute liver injury in mice. Food Chem Toxicol. (2018) 119:392-9. doi: 10.1016/j.fct.2018.01.016

60. Cheng L, Chen YH, Zhang X, Zheng XJ, Cao JX, Wu ZF, et al. A metagenomic analysis of the modulatory effect of Cyclocarya paliurus flavonoids on the intestinal microbiome in a high-fat diet-induced obesity mouse model. J Sci Food Agric. (2019) 99:3967-75. doi: 10.1002/jsfa.9622

61. Zhu KN, Jiang $\mathrm{CH}$, Tian YS, Xiao N, Wu ZF, Ma YL, et al. Two triterpeniods from Cyclocarya paliurus (Batal) Iljinsk (Juglandaceae) promote glucose uptake in 3T3-L1 adipocytes: the relationship to AMPK activation. Phytomedicine. (2015) 22:837-46. doi: 10.1016/j.phymed.2015.05.058

62. Manach C, Scalbert A, Morand C, Rémésy C, Jiménez L. Polyphenols: food sources and bioavailability. Am J Clin Nutr. (2004) 79:727-47. doi: $10.1093 /$ ajcn/79.5.727 
63. Thilakarathna SH, Rupasinghe HP. Flavonoid bioavailability and attempts for bioavailability enhancement. Nutrients. (2013) 5:3367-87. doi: 10.3390/nu5093367

64. Deng L, Lei J, He J, Liu J, Wang L, Zhang R, et al. Evaluation on genotoxicity and teratogenicity of aqueous extract from Cyclocarya paliurus leaves. Sci World J. (2014) 2014:498134. doi: 10.1155/2014/ 498134

65. Kilkenny C, Parsons N, Kadyszewski E, Festing MF, Cuthill IC, Fry D, et al. Survey of the quality of experimental design, statistical analysis and reporting of research using animals. PLoS ONE. (2009) 4:e7824. doi: 10.1371/journal.pone.0007824
Conflict of Interest: The authors declare that the research was conducted in the absence of any commercial or financial relationships that could be construed as a potential conflict of interest.

Copyright $\odot 2020 \mathrm{Liu}, \mathrm{Wu}, \mathrm{Hu}$, Qin, Guo, Wang, Wu and Liu. This is an open-access article distributed under the terms of the Creative Commons Attribution License (CC $B Y)$. The use, distribution or reproduction in other forums is permitted, provided the original author(s) and the copyright owner(s) are credited and that the original publication in this journal is cited, in accordance with accepted academic practice. No use, distribution or reproduction is permitted which does not comply with these terms. 